\title{
Prácticas culturales en vivero que influyen en la calidad de planta de Enterolobium cyclocarpum
}

\author{
Cultural practices in the nursery that affect seedling quality of Enterolobium cyclocarpum
}

\author{
Erickson Basave Villalobos a*, Miguel Á López López ${ }^{\text {b }}$, Víctor M Cetina Alcalá ${ }^{\text {b, }}$ \\ Arnulfo Aldrete ${ }^{b}$, Juan J Almaraz Suárez ${ }^{c}$ \\ *Autor de correspondencia: ${ }^{a}$ Instituto Tecnológico Superior de Venustiano Carranza, Departamento de Ingeniería Forestal, S/N, \\ Colonia El Hausteco, C.P. 73049, Venustiano Carranza, Puebla, México, tel.: 746-853-5753, erbavi_88@hotmail.com \\ ${ }^{\mathrm{b}}$ Colegio de Postgraduados, Postgrado Forestal, Texcoco, México.

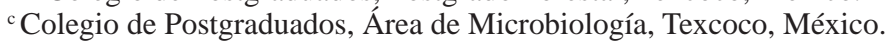

\begin{abstract}
SUMMARY
Because one of the main problems that Enterolobium cyclocarpum faces in the planting site is the presence of weeds, to grow plants presenting appropriate characteristics that enable them to overcome these adverse conditions during reforestation appears as necessary. Therefore, after evaluating the responses of various morphological and physiological indicators, and a quality test experiment, the effects of three top pruning intensities (no pruning, $25 \%$ and $50 \%$ pruning) and three fertilization regimes (traditional, exponential and conventional) were studied during nursery production. This approach was made based on the fact that top pruning and fertilization in the nursery are cultural practices that improve seedling quality as long as the pruning intensity and fertilization regime are appropriate. There was no synergy between the two practices; however, each factor influenced independently on the indicators evaluated. Top pruning at $25 \%$ and $50 \%$ did not improve the characteristics of E. cyclocarpum. However, the nutrient supply in a conventional way had a favorable effect when compared with other fertilization regimes. The results provided by the quality test established that conventional fertilization allows plants to perform satisfactorily when nutrient availability is limited.
\end{abstract}

Key words: parota, top pruning, exponential fertilization, conventional fertilization.

\section{RESUMEN}

La maleza es uno de los principales problemas que enfrenta Enterolobium cyclocarpum en el sitio de plantación; por ello, es necesario producir en vivero planta de calidad para superar esa condición adversa durante la reforestación. Prácticas como la poda aérea y fertilización en vivero han demostrado, en otras especies forestales, ser factibles para promover tal aspecto. Planteando que estas, son prácticas culturales que mejoran la calidad de planta, siempre y cuando la intensidad de poda y el régimen de fertilización sean apropiados, el objetivo de este trabajo es examinar en vivero, los efectos de tres intensidades de poda aérea (sin poda, poda al 25 \% y poda al $50 \%$ ) y de tres regímenes de fertilización (tradicional, exponencial y convencional) en planta de E. cyclocarpum. Tras la evaluación de indicadores morfológicos y fisiológicos, y de un ensayo de prueba de calidad diseñado para simular condiciones de estrés nutrimental, estas prácticas se examinaron. No hubo sinergia entre ambas prácticas; sin embargo, cada factor influyó independientemente en los indicadores evaluados. La poda aérea no favoreció la calidad de E. cyclocarpum. En cambio, el suministro de nutrimentos en forma convencional sí tuvo un efecto favorable. Adicionalmente, los resultados aportados por el ensayo de prueba de calidad establecen que las dosis suministradas de fertilizante $\left(140 \mathrm{mg} \mathrm{L}^{-1}\right)$ mediante el régimen convencional generan en las plantas reservas nutrimentales suficientes para que estas se desempeñen adecuadamente ante un ambiente controlado de estrés nutrimental; no obstante, tras dar pautas iniciales, se recomiendan trabajos en campo para dilucidar estas respuestas.

Palabras clave: parota, poda aérea, fertilización exponencial, fertilización convencional.

\section{INTRODUCCIÓN}

Durante la última década, en México y Centroamérica, el establecimiento de árboles multipropósito en terrenos ganaderos o agrícolas se ha incrementado debido a los beneficios ambientales y económicos que estos aportan. En este contexto, la parota (Enterolobium cyclocarpum (Jacq.) Griseb.) es una de las especies más demandadas para reforestar en fincas que buscan incrementar su renta- bilidad mediante la inclusión de especies maderables con alto valor comercial y ecológico (Ibrahim y Zapata 2012).

Sin embargo, a menudo las reforestaciones con esta especie, con planta producida en vivero, enfrentan diversas condiciones adversas en el sitio de plantación que afectan el desempeño de las plantas. La presencia de malezas es uno de los factores más importantes que limitan la disponibilidad de luz, agua y nutrientes. Para solucionar este problema, es necesario realizar deshierbes, los cuales deben 
ser meticulosos y frecuentes. No obstante, el cumplimento de estas condiciones incrementa considerablemente los costos de operación (Ibrahim y Zapata 2012).

Enfocados en solucionar el mismo problema, pero en otras especies forestales y en otras regiones, Jobidon et al. (2003) demostraron que con el uso de planta competitiva producida en vivero, se logran tasas favorables de supervivencia y crecimiento sin necesidad de deshierbes meticulosos y frecuentes. Una planta de vivero es competitiva cuando, por sus características morfológicas y fisiológicas, se adapta rápidamente en el sitio de plantación (Grossnickle 2012). Villar-Salvador et al. (2012) argumentan que sí en vivero se produce planta vigorosa, con capacidad fotosintética elevada y con contenidos altos de reservas nutrimentales, se satisface esta condición.

Si se utiliza germoplasma de calidad y se aplican prácticas culturales adecuadas, es posible que en un ciclo normal de producción se obtengan plantas con las características mencionadas. Desafortunadamente, debido a la poca atención que ha recibido E. cyclocarpum en este aspecto, las prácticas culturales que promueven tales características se desconocen o son poco comprendidas. Ejemplos de ellas, son la poda aérea y la fertilización. La poda aérea como práctica cultural para incrementar la calidad de planta de vivero no es común. No obstante, Cetina et al. $(1999,2002)$ han demostrado con plantas de Pinus greggii Engelm. que si esta práctica se emplea adecuadamente, es decir, con una intensidad apropiada, se mejora el crecimiento de las plantas en vivero y campo, por los efectos fisiológicos asociados con la remoción de la dominancia o control apical (Wilson 2000). Sin embargo, en otras especies como Nothofagus nervosa, la respuesta no ha sido la misma (Donoso et al. 2009).

La fertilización en vivero sí es una práctica ampliamente usada, y su eficacia depende, en parte, del régimen implementado. De acuerdo con Oliet et al. (2013) y VillarSalvador et al. (2013), un régimen ideal de fertilización debe satisfacer las demandas del crecimiento, pero también debe promover la existencia de contenidos altos de reservas nutrimentales para que las plantas sean capaces de desempeñarse satisfactoriamente, aun cuando la disponibilidad de nutrimentos en el sitio de plantación sea limitada. Para E. cyclocarpum estas reservas son importantes mientras se adapta (aclimata) al terreno y logra fijar nitrógeno. Timmer (1997) señala que sólo a través de un consumo de lujo, es decir, mediante el suministro de fertilizante en exceso sin causar toxicidad, la planta logra acumular dichas reservas y que el régimen de fertilización más apropiado para inducir tal consumo, es el exponencial. Sin embargo, Oliet et al. (2013) indican que debido a los diferentes patrones de crecimiento entre especies, el régimen exponencial no es factible en todos los casos.

Si se considera que los efectos favorables de estas prácticas sobre la calidad de planta están en función de la especie, de la intensidad de poda aérea y del régimen de fertilización implementado, es importante estudiar detalladamente sus efectos. Por ello, el objetivo del presente trabajo es estudiar el efecto de dichas prácticas en plantas de E. cyclocarpum mediante la evaluación de diversos indicadores morfológicos y fisiológicos de calidad, y de un ensayo de prueba de calidad.

\section{MÉTODOS}

Las fases experimentales involucradas se realizaron en condiciones controladas de invernadero, donde la temperatura, el fotoperiodo y la humedad relativa fueron de $30^{\circ} \mathrm{C}$ día y $15^{\circ} \mathrm{C}$ noche, $16 \mathrm{~h}$ (iluminancia de 1.000 lx generada con lámparas incandescentes), y 70-85 \%, respectivamente. El invernadero donde se instaló el experimento se localiza en el vivero del Postgrado Forestal del Colegio de Postgraduados Campus Montecillo, Texcoco, Estado de México

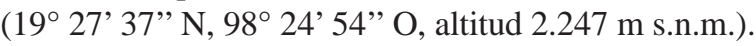

Producción de planta. Durante marzo de 2012 se recolectó semilla de E. cyclocarpum en rodales naturales de la región de Tierra Caliente del estado de Guerrero, México. Posteriormente, tras haber sido almacenada en un banco de germoplasma forestal a $5{ }^{\circ} \mathrm{C}$, con un contenido de humedad del $8 \%$ al $11 \%$, en noviembre de 2012 se usaron 700 semillas uniformes en tamaño que fueron previamente escarificadas con una lija y puestas en remojo durante $24 \mathrm{~h}$ en agua corriente para sembrarse directamente en contenedores de plástico de $380 \mathrm{~mL}$ llenados con sustrato compuesto por turba de musgo (peat moss), perlita y vermiculita en proporciones 2:1:1. Se sembró una semilla por contenedor.

Tratamientos aplicados y diseño experimental. Tres semanas después de la siembra (07 de diciembre de 2012), del lote producido, se seleccionaron 432 plantas con base en criterios de uniformidad en altura $(9-10 \mathrm{~cm})$. Entonces, se les aplicaron los siguientes tratamientos o niveles de poda aérea y fertilización.

Los tratamientos de poda, expresados como intensidad, fueron tres: sin poda o poda al $0 \%$, poda al $25 \%$ y poda al $50 \%$. Estos consistieron en la remoción de la fracción de biomasa aérea correspondiente a la intensidad, para lo cual se tomó como referencia la longitud entre la primera hoja y el ápice del vástago. Dichos tratamientos se aplicaron solo una vez junto con la primera fertilización.

Los tratamientos de fertilización, referidos como regímenes, fueron tres: régimen tradicional $(\mathrm{T})$, régimen exponencial (EXP) y régimen convencional (CO). Con base en lo sugerido por Aldana y Aguilera (2003), tales tratamientos se aplicaron dos veces por semana durante un periodo de 10 semanas; esto, con el fin de cubrir las tres principales fases de crecimiento en las que un cultivo tropical cuya edad apta para establecerse en terreno oscila entre los 3-4 meses, demanda fertilizante.

El régimen tradicional de fertilización representó el programa de fertilización mediante el cual se producen especies forestales tropicales en contenedor para las reforestaciones en México. Este programa emplea como fuente 
de nutrimentos fertilizantes hidrosolubles Peters ${ }^{\circledR}$, específicos para cada fase de crecimiento de la planta (Aldana y Aguilera 2003) (cuadro 1).

El régimen exponencial de fertilización se diseñó para incrementar la adición de nutrimentos en forma exponencial en cada fecha de aplicación. Los cálculos se basaron en una función exponencial descrita por Timmer (1997) (ecuación 1):

$$
N_{T}=N_{S}\left(\mathrm{e}^{r t}-1\right)-N_{(\mathrm{t}-1)}
$$

Donde: $t=10$, es la duración del periodo de crecimiento en semanas; $N_{T}$ es la cantidad de fertilizante aplicado en un día específico t; $N_{\mathrm{t}-1}$ es la cantidad acumulativa de fertilizante suministrado en un tiempo específico (incluyendo la aplicación previa), y $N_{\mathrm{S}}$ es el contenido inicial de nitrógeno en plantas recién germinadas (9,72 $\left.\mathrm{mg}^{\text {planta }}{ }^{-1}\right)$. $N_{S}$ se determinó mediante el análisis de una muestra compuesta por 50 plantas recién germinadas.

El factor $r$ es la tasa de adición relativa de nutrimentos para incrementar $N_{S}$ al nivel final de $\left(N_{T}+N_{S}\right)$ el cual se determinó por la función derivada de la ecuación 2 :

$$
r=\left(\ln \left[N_{T} / N_{S}+1\right]\left[\mathrm{e}^{r t}-1\right] / \chi\right)
$$

Donde: $N_{T}$ es la cantidad óptima de fertilizante necesario para alcanzar los requerimientos de las plantas.

Por la escasa información sobre aspectos nutrimentales en esta especie; $N_{T}\left(25,40 \mathrm{mg}\right.$ planta $\left.^{-1}\right)$ se determinó a partir de la cantidad de nitrógeno aplicado por planta en el programa de fertilización tradicional. Se programaron dos aplicaciones semanales durante las 10 semanas del periodo de crecimiento, entonces $\chi$ (número de aplicaciones) $=20$. Las dosis obtenidas (mg planta-1) en cada aplicación se multiplicaron por 24. Dicha cifra corresponde a la cantidad de plantas fertilizadas por litro de solución nutritiva en todos los tratamientos. Con estos valores, se determinaron las concentraciones de nitrógeno $\left(\mathrm{mg} \mathrm{L}^{-1}\right)$ de nutrimentos en la solución nutritiva. Las concentraciones específicas de nitrógeno aplicado se muestran en la figura 1. Para todas las aplicaciones del exponencial se utilizó fertilizante hidrosoluble Peters Professional ${ }^{\circledR}$ General Purpose 20-20-20 ${ }^{\mathrm{TM}}$.
El régimen convencional de fertilización involucró la aplicación de dosis iguales $\left(140 \mathrm{mg} \mathrm{L}^{-1}\right)$ en las 20 fertilizaciones realizadas a lo largo de las diez semanas. Esta dosis se derivó de los cálculos obtenidos en el régimen exponencial (figura 1). De igual manera que en el tratamiento exponencial, la fuente de nutrimentos fue Peters Professional ${ }^{\circledR}$ General Purpose 20-20-20 ${ }^{\mathrm{TM}}$.

Los fertilizantes aplicados fueron la única fuente de nutrimentos, pues en todas las aplicaciones y riegos se utilizó agua destilada. El pH (5,5-6,5) y la conductividad eléctrica (1.200-1.800 $\left.\mu \mathrm{S} \mathrm{cm}^{-1}\right)$ se mantuvieron en los rangos óptimos recomendados por Landis (1989).

En el experimento se utilizó un arreglo factorial 3x3 para el diseño de los tratamientos. Las nueve combinaciones de tratamientos resultantes de los dos factores estudiados, se replicaron cuatro veces y se asignaron al azar mediante un diseño completamente aleatorizado a las unidades experimentales (36) conformadas por 12 plantas.

Variables evaluadas. Tres semanas después de la última fertilización (10 de marzo de 2013), se tomaron al azar 12 plantas por tratamiento, a las cuales se les evaluaron diver-

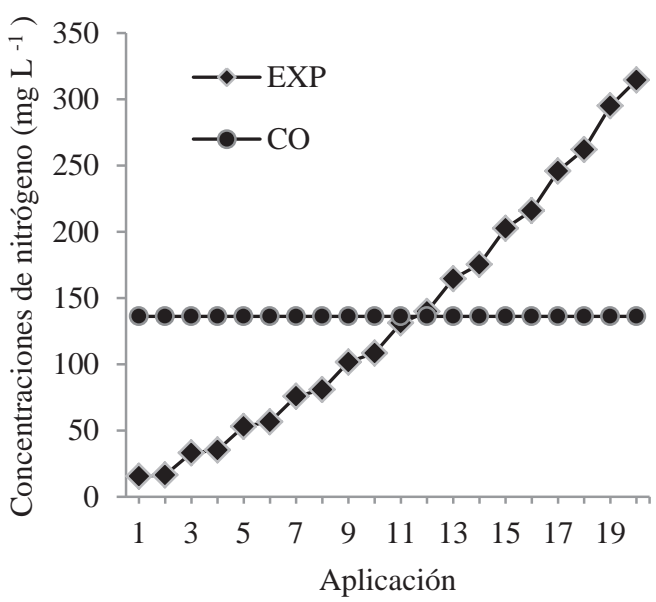

Figura 1. Concentraciones de nitrógeno suministrado en cada aplicación de los regímenes de fertilización exponencial (EXP) y convencional (CO).

Concentrations of nitrogen applied in each application of exponential (EXP) and conventional (CO) fertilization regimes.

Cuadro 1. Programa de fertilización empleado en el tratamiento de fertilización tradicional.

Rates of fertilizer applied with the traditional fertilization treatment.

\begin{tabular}{lccc}
\hline \multicolumn{1}{c}{ Fase de crecimiento } & $\begin{array}{c}\text { Duración } \\
\text { (semanas) }\end{array}$ & $\begin{array}{c}\text { Fertilizante empleado } \\
\text { (fórmula) }\end{array}$ & $\begin{array}{c}\text { Dosis de nitrógeno } \\
\left(\mathrm{mg} \mathrm{L}^{-1}\right)\end{array}$ \\
\hline Establecimiento & 3 & $9 \mathrm{~N}: 45 \mathrm{P}_{2} \mathrm{O}_{5}: 15 \mathrm{~K}_{2} \mathrm{O}$ & 25 \\
Crecimiento rápido & 4 & $20 \mathrm{~N}: 10 \mathrm{P}_{2} \mathrm{O}_{5}: 20 \mathrm{~K}_{2} \mathrm{O}$ & 50 \\
Endurecimiento & 3 & $4 \mathrm{~N}: 25 \mathrm{P}_{2} \mathrm{O}_{5}: 35 \mathrm{~K}_{2} \mathrm{O}$ & 50 \\
\hline
\end{tabular}


sas características morfológicas y fisiológicas relacionadas con indicadores de calidad de planta. Por el lado de las características morfológicas, de acuerdo con la metodología empleada por Cetina et al. (1999), se midió la altura de la parte aérea $(\mathrm{cm})$, el diámetro del tallo a la altura del cuello de la raíz (mm), el peso seco de la parte aérea (g) y el peso seco de la parte radical (g). Con las observaciones de altura de la parte aérea y diámetro a la altura del cuello, se determinó el índice de robustez (IR); cociente que resulta al dividir estos dos componentes (Johnson y Cline 1991), mientras que con los valores de los pesos secos tanto de la parte aérea como radical se calculó la relación parte aérearaíz (R: PSA/PSR) (Johnson y Cline 1991). Finalmente, el índice de calidad de Dickson et al. se obtuvo siguiendo la siguiente ecuación (Johnson y Cline 1991) (3):

Índice de calidad de Dickson et al.

$$
=\frac{\text { Biomasa total seca }(\mathrm{g})}{\frac{\text { Altura }(\mathrm{cm})}{\text { Diámetro }(\mathrm{mm})}+\frac{\text { Peso seco aéreo }(\mathrm{g})}{\text { Peso seco radical }(\mathrm{g})}}
$$

Con relación con los indicadores fisiológicos de calidad, se evaluó la tasa de fotosíntesis neta (TFN [ $\mu \mathrm{mol}$ $\left.\mathrm{m}^{-2} \mathrm{~s}^{-1}\right]$ ), el contenido de clorofila (unidades SPAD) y el contenido de nitrógeno, fósforo y potasio. La tasa de fotosíntesis neta se midió con un CIRAS -2 (PP Systems, Haverhill, Ma, EE. UU.) de 09:00 - 12:00 h y el contenido de clorofila se determinó indirectamente con un medidor de clorofila portátil Minolta SPAD 502 Plus (Spectum Technologies Inc., Plainfield, IL. EE. UU.), el contenido de nitrógeno, fósforo y potasio se obtuvo con los datos registrados de biomasa y concentraciones de dichos nutrimentos. Las concentraciones de los nutrimentos se determinaron en muestras compuestas de tejido vegetal (planta completa). El nitrógeno se determinó por el método de micro-kjeldahl, el fósforo mediante fotoclorimetría por reducción con molibdato-vanadato y el potasio a través de espectrofotometría de emisión de flama.

Ensayo de prueba de calidad en tezontle. Posteriormente, considerando que en un ambiente de campo con competencia interespecífica ante malezas, la disponibilidad de nutrientes es limitada; se diseñó y se estableció en marzo de 2013, un ensayo para simular condiciones de estrés nutrimental y así evaluar la calidad de las plantas en función de las reservas nutrimentales. Para este fin, se construyó una cama de $30 \mathrm{~cm}$ de profundidad con sustrato de tezontle rojo (escoria volcánica) para garantizar que la disponibilidad de nutrimentos fuera escasa o nula. Se utilizaron seis plantas por tratamiento elegidas al azar y se colocaron en la cama en hileras teniendo un total de 54 plantas bajo un diseño experimental completamente aleatorizado.

El ensayo finalizó en mayo de 2013. Durante ese lapso (tres meses), las plantas se regaron frecuentemente con agua destilada para evitar el suministro externo de nutrimentos. Se efectuaron dos mediciones de altura $(\mathrm{cm}) \mathrm{y}$ diámetro del tallo (mm); una al inicio y la otra al final del ensayo. Con los valores obtenidos se calcularon las tasas de crecimiento absoluto mensual (TCA) con base en la siguiente ecuación (4):

$$
\mathrm{TCA}=\left(\frac{\mathrm{T} 2-\mathrm{T} 1}{\Delta T}\right)
$$

Donde: $\mathrm{T}_{1} \mathrm{y} \mathrm{T}_{2}$ son las variables medidas en el tiempo de la primera y segunda evaluación, respectivamente, y $\Delta t$ es el intervalo de tiempo entre las dos mediciones.

Análisis estadístico. Para comparar los efectos de los factores y sus interacciones en los indicadores morfológicos y fisiológicos de calidad, se realizó un análisis de varianza (ANDEVA) considerando el cumplimiento de los supuestos de normalidad y homogeneidad de varianzas. Los efectos se consideraron estadísticamente significativos cuando el valor de $P<0,05$. Cuando los efectos resultaron significativos, las diferencias estadísticas entre medias se identificaron mediante la prueba de diferencia significativa honesta (DSH) de Tukey $(\alpha=0,05)$. El análisis estadístico de los datos se hizo con el software InfoStat (Di Rienzo et al. 2012). En el caso del contenido de nitrógeno, fósforo y potasio no se realizó análisis estadístico, únicamente se reportan los valores promedio por muestra compuesta. Adicionalmente, debido a que la prueba de calidad se diseñó especialmente para simular condiciones de baja fertilidad y por consiguiente evaluar el desempeño de las plantas en virtud de sus reservas nutrimentales, las tasas de crecimiento absoluto mensual se analizaron por tratamiento y no por factores, de esa manera se determinó la influencia de las reservas nutrimentales sobre el crecimiento de las plantas.

\section{RESULTADOS}

Indicadores morfológicos y fisiológicos de calidad. Al finalizar el periodo de crecimiento en vivero, en los indicadores morfológicos y fisiológicos de calidad evaluados, no se presentaron efectos por la interacción entre la poda aérea y el régimen de fertilización; no obstante, existió influencia independiente de los factores estudiados (cuadro 2).

Mientras que la poda aérea influyó únicamente sobre los atributos morfológicos (con excepción del índice de calidad de Dickson et al.); el régimen de fertilización tuvo efectos en la mayoría de las variables, excepto en la tasa de fotosíntesis neta (cuadro 2). A pesar de la discrepancia entre el número de variables afectadas por cada factor, se destaca que en la mayoría de las variables los efectos ejercidos fueron altamente significativos (cuadro 2).

Por parte del factor poda aérea, los valores más elevados en las variables morfológicas ocurrieron con el tratamiento sin poda (cuadro 3). En cambio, los valores más altos de las variables relacionadas al régimen de fertilización se encontraron con el tratamiento de fertilización convencional (cuadro 4). 
Cuadro 2. Resultados de los análisis de varianza para los indicadores morfológicos y fisiológicos de calidad evaluados en plantas de Enterolobium cyclocarpum.

Summary of the variance analysis for top pruning and fertilization regime effects on morphological and physiological indicators of quality in Enterolobium cyclocarpum seedlings.

\begin{tabular}{|c|c|c|c|c|c|c|c|c|c|}
\hline \multirow{2}{*}{$\begin{array}{l}\text { Fuente de } \\
\text { variación }\end{array}$} & $\mathrm{AL}^{1}$ & $\mathrm{DM}^{2}$ & $\mathrm{PSA}^{3}$ & $\mathrm{PSR}^{4}$ & R:PSA/PSR ${ }^{5}$ & $\mathrm{IR}^{6}$ & $\mathrm{ICD}^{7}$ & $\mathrm{TFN}^{8}$ & $\mathrm{CC}^{9}$ \\
\hline & \multicolumn{9}{|c|}{ Valor $P(\alpha=0,05)$} \\
\hline Poda aérea & $<0,001^{* * *}$ & $0,001^{* *}$ & $<0,001^{* * *}$ & $<0,001^{* * *}$ & $0,044^{*}$ & $<0,001^{* * *}$ & $0,059^{\text {ns }}$ & $0,839^{\text {ns }}$ & $0,208^{\mathrm{ns}}$ \\
\hline R.F $\rrbracket$ & $<0,001^{* * *}$ & $0,003^{* *}$ & $<0,001^{* * *}$ & $0,001^{* *}$ & $<0,001^{* * *}$ & $0,001^{* *}$ & $0,003^{* *}$ & $0,173^{\mathrm{ns}}$ & $0,045^{*}$ \\
\hline Interacción & $0,253^{\text {ns }}$ & $0,555^{\mathrm{ns}}$ & $0,352^{\text {ns }}$ & $0,606^{\text {ns }}$ & $0,404^{\mathrm{ns}}$ & $0,208^{\text {ns }}$ & $0,927^{\mathrm{ns}}$ & $0,188^{\mathrm{ns}}$ & $0,251^{\mathrm{ns}}$ \\
\hline
\end{tabular}

ๆ: régimen de fertilización. 1: altura; 2: diámetro; 3: peso seco aéreo; 4: peso seco radical; 5: relación peso seco aéreo -peso seco radical; 6: índice de robustez; 7: índice de calidad de Dickson et al.; 8: tasa de fotosíntesis neta; 9: contenido de clorofila.

$*^{* * *},{ }^{* * *}$ : significativos a los niveles de 0,05, 0,01 y 0,001 de probabilidad, respectivamente. ns: no significativo. Fertilization regime. 1: Shoot height; 2: Root collar diameter; 3: Shoot dry weight; 4: Root dry weight; 5: Shoot-root ratio; 6: Sturdiness index; 7: Dickson’s quality index; 8: Net photosynthesis rate; 9: Chlorophyll content. ${ }^{* * *}{ }^{*},{ }^{* *}$ : Significant to the levels of $0.05,0.01$ and 0.001 of probability, respectively. ns: Not significant.

Cuadro 3. Valores promedio de los indicadores morfológicos de calidad evaluados en plantas de Enterolobium cyclocarpum en respuesta a los tratamientos de poda aérea.

Average values of morphological indicators of quality assessed in Enterolobium cyclocarpum seedlings in response to top pruning treatments.

\begin{tabular}{lccc}
\hline \multirow{2}{*}{ Variables morfológicas } & \multicolumn{3}{c}{ Intensidad de poda aérea } \\
\cline { 2 - 3 } & Sin poda & Poda al $25 \%$ & Poda al $50 \%$ \\
\hline Altura $(\mathrm{cm})$ & $28,5 \pm 0,47 \mathrm{a}^{\text {q⿻ }}$ & $21,0 \pm 0,47 \mathrm{c}$ & $22,5 \pm 0,47 \mathrm{~b}$ \\
Diámetro (mm) & $4,4 \pm 0,05 \mathrm{a}$ & $4,1 \pm 0,05 \mathrm{~b}$ & $4,3 \pm 0,05 \mathrm{a}$ \\
Peso seco aéreo (g) & $1,40 \pm 0,03 \mathrm{a}$ & $1,05 \pm 0,03 \mathrm{~b}$ & $1,10 \pm 0,03 \mathrm{~b}$ \\
Peso seco radical (g) & $0,77 \pm 0,02 \mathrm{a}$ & $0,60 \pm 0,02 \mathrm{~b}$ & $0,61 \pm 0,02 \mathrm{~b}$ \\
Índice de robustez & $6,47 \pm 0.12 \mathrm{a}$ & $5,13 \pm 0,12 \mathrm{~b}$ & $5,28 \pm 0,12 \mathrm{~b}$ \\
R: PSA/PSR & $1,83 \pm 0,03 \mathrm{a}$ & $1,75 \pm 0,03 \mathrm{a}$ & $1,80 \pm 0,03 \mathrm{a}$ \\
\hline
\end{tabular}

ๆ: relación peso seco aéreo-peso seco radical. " "De acuerdo con DSH de Tukey $\alpha=0,05$, medias con letras iguales dentro de la misma fila no son significativamente diferentes $(P>0,05)$.

"Shoot - root ratio. "According to Tukey's LSD $\alpha=0.05$, means ( \pm EE) with the same letter within the same line are not significantly different $(P>0.05)$.

Contenido de nitrógeno, fósforo y potasio. En comparación con las plantas sometidas a la combinación de tratamientos sin poda + fertilización tradicional (testigo), las plantas que recibieron la combinación de tratamientos sin poda + fertilización convencional presentaron los contenidos más altos de nitrógeno y fósforo con incrementos que oscilaron en 119 \% y 12 \%, respectivamente. Referente al potasio, el contenido más elevado se manifestó en plantas de la combinación de factores testigo (cuadro 5).

Ensayo de prueba de calidad en tezontle. Cuando las plantas se sometieron a condiciones de estrés nutrimental mediante el ensayo de prueba de calidad en tezontle no se afectó su supervivencia (variable evaluada pero no presentada). En cambio, existieron diferencias altamente significativas referente con las tasas de crecimiento absoluto mensual en altura y diámetro ( $P \leq 0,0001$ y $P=0,0002$, respectivamente). Las plantas que recibieron la combinación de tratamientos sin poda + fertilización exponencial, destacaron con su crecimiento en altura, pues en comparación con las plantas de los tratamientos testigo, casi triplicaron mensualmente la tasa de crecimiento absoluto (cuadro 6). Sin embargo, no sucedió lo mismo en el crecimiento en diámetro, ya que en este caso las plantas de la combinación de tratamientos sin poda + fertilización convencional, crecieron 1,5 veces más por mes en relación con las del testigo (cuadro 6). 
Cuadro 4. Valores promedio de los indicadores morfológicos y fisiológicos de calidad evaluados en plantas de Enterolobium cyclocarpum en respuesta a los regímenes de fertilización.

Average values of morphological and physiological indicators of quality assessed in Enterolobium cyclocarpum seedlings in response to fertilization treatments.

\begin{tabular}{|c|c|c|c|c|c|c|}
\hline \multirow{2}{*}{ Variables } & \multicolumn{6}{|c|}{ Régimen de fertilización } \\
\hline & \multicolumn{2}{|c|}{ Tradicional } & \multicolumn{2}{|c|}{ Convencional } & \multicolumn{2}{|c|}{ Exponencial } \\
\hline \multicolumn{7}{|l|}{ Morfológicas } \\
\hline Altura $(\mathrm{cm})$ & \multicolumn{2}{|c|}{$22,2 \pm 0,47 \mathrm{~b}^{\dagger}$} & \multicolumn{2}{|c|}{$25,5 \pm 0,47 a$} & \multicolumn{2}{|c|}{$24,3 \pm 0,47 a$} \\
\hline Diámetro (mm) & \multicolumn{2}{|c|}{$4,3 \pm 0,05 \mathrm{ab}$} & \multicolumn{2}{|c|}{$4,4 \pm 0,05 a$} & \multicolumn{2}{|c|}{$4,2 \pm 0,05 b$} \\
\hline Peso seco aéreo (g) & \multicolumn{2}{|c|}{$1,20 \pm 0,03 b$} & \multicolumn{2}{|c|}{$1,34 \pm 0,03 a$} & \multicolumn{2}{|c|}{$1,02 \pm 0,03 c$} \\
\hline Peso seco radical (g) & \multicolumn{2}{|c|}{$0,63 \pm 0,02 b$} & \multicolumn{2}{|c|}{$0,71 \pm 0,02 a$} & \multicolumn{2}{|c|}{$0,64 \pm 0,02 b$} \\
\hline Índice de robustez & \multicolumn{2}{|c|}{$5,21 \pm 0,12 b$} & \multicolumn{2}{|c|}{$5,81 \pm 0,12 \mathrm{a}$} & \multicolumn{2}{|c|}{$5,86 \pm 0,12 \mathrm{a}$} \\
\hline ICD & \multicolumn{2}{|c|}{$0,24 \pm 0,01 b$} & \multicolumn{2}{|c|}{$0,27 \pm 0,01 \mathrm{a}$} & \multicolumn{2}{|c|}{$0,24 \pm 0,01 b$} \\
\hline $\mathrm{R}: \mathrm{PSA} / \mathrm{PSR}^{\text {ศा }}$ & \multicolumn{2}{|c|}{$1,61 \pm 0,03 b$} & \multicolumn{2}{|c|}{$1,89 \pm 0,03 a$} & \multicolumn{2}{|c|}{$1,89 \pm 0,03 a$} \\
\hline \multicolumn{7}{|l|}{ Fisiológicas } \\
\hline CC (unidades SPAD) भศศ & \multicolumn{2}{|c|}{$28,36 \pm 1,00 b$} & \multicolumn{2}{|c|}{$31,30 \pm 1,00 \mathrm{a}$} & \multicolumn{2}{|c|}{$28,08 \pm 1,00 b$} \\
\hline \multirow{2}{*}{\multicolumn{7}{|c|}{ 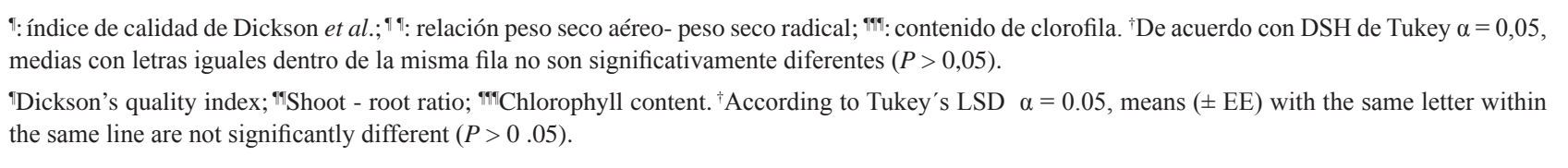 }} \\
\hline & & & & & & \\
\hline Cuadro 5. Concentración y conte & de nitróger & sforo y p & en respue & tratamiento & poda y fer & ión. \\
\hline & nitrogen, pho & us, and pot & in respon & runing and $\mathrm{f}$ & ation treatm & \\
\hline & & ntración ( & & & ido (mg p & \\
\hline & Nitrógeno & Fósforo & Potasio & Nitrógeno & Fósforo & Potasio \\
\hline $\mathrm{T} 1=$ Sin poda $+\mathrm{T}$ & 13,2 & 10,9 & 23,9 & 25,98 & 21,14 & 47,04 \\
\hline $\mathrm{T} 2=$ Poda al $25 \%+\mathrm{T}$ & 24,4 & 13,0 & 22,3 & 37,05 & 19,74 & 33,86 \\
\hline $\mathrm{T} 3=$ Poda al $50 \%+\mathrm{T}$ & 20,3 & 11,6 & 20,6 & 29,42 & 16,81 & 29,85 \\
\hline $\mathrm{T} 4=$ Sin poda $+\mathrm{CO}$ & 23,3 & 09,8 & 12,2 & 56,75 & 23,54 & 29,71 \\
\hline T5= Poda al $25 \%+$ CO & 12,2 & 08,4 & 16,3 & 22,27 & 15,33 & 29,76 \\
\hline $\mathrm{T} 6=$ Poda al $50 \%+\mathrm{CO}$ & 27,4 & 08,4 & 12,5 & 52,18 & 15,99 & 23,80 \\
\hline $\mathrm{T} 7=$ Sin poda $+\mathrm{EXP}$ & 23,3 & 09,2 & 17,9 & 49,08 & 19,38 & 37,70 \\
\hline T8= Poda al 25\% + EXP & 24,4 & 09,0 & 19,1 & 39,19 & 14,45 & 30,67 \\
\hline $\mathrm{T} 9=$ Poda al 50\% + EXP & 29,4 & 08,1 & 17,6 & 52,59 & 14,48 & 31,48 \\
\hline
\end{tabular}

T: régimen de fertilización tradicional; CO: régimen de fertilización convencional; EXP: régimen de fertilización exponencial.

${ }^{1}$ Nitrogen; ${ }^{2}$ Phosphorus; ${ }^{3}$ Potassium. T: Traditional fertilization regime; CO: Conventional fertilization regime; EXP: Exponential fertilization regime. 
Cuadro 6. Tasas promedio de crecimiento absoluto en plantas de Enterolobium cyclocarpum plantadas en condiciones de escasa o nula disponibilidad de nutrientes.

Absolute growth rates in Enterolobium cyclocarpum seedlings planted on low availability of nutrients.

\begin{tabular}{|c|c|c|}
\hline \multirow{2}{*}{ Combinación de tratamientos } & \multicolumn{2}{|c|}{ Tasas de crecimiento absoluto } \\
\hline & Altura (cm mes $\left.{ }^{-1}\right)$ & Diámetro $\left(\mathrm{mm} \mathrm{mes}^{-1}\right)$ \\
\hline $\mathrm{T} 1=$ Sin poda $+\mathrm{T}$ & $1,2 \pm 0,55 b c$ & $0,4 \pm 0,02 \mathrm{ab}$ \\
\hline $\mathrm{T} 2=$ Poda al $25 \%+\mathrm{T}$ & $0,9 \pm 0,55 c$ & $0,2 \pm 0,02 b$ \\
\hline $\mathrm{T} 3=$ Poda al $50 \%+\mathrm{T}$ & $1,0 \pm 0,55 c$ & $0,2 \pm 0,02 b$ \\
\hline $\mathrm{T} 4=\mathrm{Sin}$ poda $+\mathrm{CO}$ & $2,5 \pm 0,55 a b$ & $0,6 \pm 0,02 \mathrm{a}$ \\
\hline T5= Poda al 25\% + CO & $2,8 \pm 0,55 a$ & $0,5 \pm 0,02 \mathrm{ab}$ \\
\hline T6= Poda al 50\% + CO & $3,0 \pm 0,55 a$ & $0,6 \pm 0,02 \mathrm{a}$ \\
\hline $\mathrm{T} 7=$ Sin poda + EXP & $3,3 \pm 0,55 a$ & $0,5 \pm 0,02 \mathrm{ab}$ \\
\hline T8= Poda al 25\% + EXP & $2,8 \pm 0,55 a$ & $0,5 \pm 0,02 \mathrm{ab}$ \\
\hline T9= Poda al 50\% + EXP & $3,2 \pm 0,55 a$ & $0,5 \pm 0,02 \mathrm{ab}$ \\
\hline
\end{tabular}

T: régimen de fertilización tradicional; CO: régimen de fertilización convencional; EXP: régimen de fertilización exponencial. ๆ De acuerdo con DMHS de Tukey $\alpha=0,05$, medias con letras iguales dentro de la misma columna no son significativamente diferentes $(P>0,05)$.

T: Traditional fertilization regime; CO: Conventional fertilization regime; EXP: Exponential fertilization regime. " According to Tukey's LSD $\alpha=$ 0.05 , means $( \pm \mathrm{EE})$ with the same letter within the same column are not significantly different $(P>0.05)$.

\section{DISCUSIÓN}

Poda aérea. Con base en los resultados obtenidos, se carece de evidencia para definir si la poda aérea promueve en Enterolobium cyclocarpum características apropiadas para desempeñarse satisfactoriamente en sitios competitivos. Cuando se podó el 25 \% de la biomasa aérea, las plantas respondieron favorablemente al índice de robustez y a la relación peso seco aéreo-peso seco radical, los cuales asocian a valores bajos con mejor calidad; sin embargo, los valores más elevados en altura, diámetro, peso seco aéreo y radical correspondieron a las plantas sin podar (cuadro 4).

Cuando Cetina et al. (1999) podaron planta de Pinus greggii empleando las mismas intensidades probadas en este estudio, reportaron un efecto similar al ocurrido en $E$. cyclocarpum, el crecimiento en altura y diámetro se redujo; sin embargo, en la biomasa de la parte aérea y radical se estimuló el crecimiento, principalmente en las plantas donde se removió el 50 \% de su biomasa aérea. En las variables de biomasa total, radical y aérea, los resultados de ambos estudios son diferentes, ya que en este estudio la biomasa producida fue menor en las plantas podadas. Según estos autores, en $P$. greggii los aumentos en biomasa aérea y radical se debieron a que esta práctica incrementó la formación y el crecimiento de más ramas laterales con su respectiva área foliar, efecto que está en concordancia con lo establecido por Wilson (2000), pero no con lo re- portado por Hawkins y Henry (1999), quienes al analizar esta práctica en plantas de Thuja plicata Donn ex. D. Don encontraron que la poda no favorece la acumulación de biomasa en dicha especie. Sin embargo, el enfoque de podas implementado por Hawkins y Henry (1999) es distinto al de Cetina et al. (1999) y al de este trabajo.

Comparando los resultados obtenidos por Cetina et al. (1999) con los de este trabajo, hipotéticamente, existen dos razones por las cuales E. cyclocarpum no respondió positivamente a la poda. En primer lugar, se atribuye a las diferencias entre especies, ya que varían los patrones de crecimiento y ramificación entre una especie conífera y otra latifolia (Cline y Harrington 2007). Tal hipótesis también es sustentada por Donoso et al. (2009), pues al evaluar el efecto de dicha práctica sobre la calidad de planta de Nothofagus nervosa, estos autores, tampoco encontraron una respuesta favorable. En segundo lugar influyó la edad de las plantas, pues cuando los individuos de $P$. greggii se podaron, estos tenían seis meses de edad; en cambio, los de E. cyclocarpum solo tenían un mes. Posiblemente, entre menor sea la edad de la plantas, estas son más vulnerables al estrés que esta práctica ocasiona por la remoción de biomasa. Si el estrés ocasionado no provoca que las plantas mueran, estas demandarán grandes cantidades de recursos para recuperarse mediante el rebrote. Para rebrotar, las plantas canalizan recursos hacia el almacenamiento para formar las reservas necesarias en dicho proce- 
so (Berendse et al. 2007). No obstante, los mismos autores puntualizan que la formación de reservas compite directamente por recursos con el crecimiento, originando que entre más altas sean las demandas, el crecimiento se afecte en mayor medida. A pesar de esto, esta hipótesis es relativamente coherente porque cuando Donoso et al. (2009) podaron sus plantas, estas no tenían una edad vulnerable y tampoco hubo una respuesta positiva por las podas.

A pesar de que la capacidad de crecimiento y acumulación de biomasa en E. cyclocarpum se vio afectada por las podas, tal limitación permitió que las plantas tuvieran un crecimiento más equilibrado, pues como se indicó al principio, las plantas que recibieron poda al $25 \%$ fueron más robustas y tuvieron una mejor relación entre la parte aérea y radical (cuadro 2). Tal efecto, tiene implicaciones para un mejor manejo, ya que a menudo la poda aérea se usa como una herramienta correctiva para uniformizar los lotes de planta cuando estos exhiben heterogeneidad en tamaño por un manejo inapropiado durante el periodo de producción, lo cual tiene un efecto contraproducente sobre las plantas en el lugar de plantación (Mc Nabb y Vanderschaaf 2005).

Régimen de fertilización. A pesar de que diversos estudios en latifolias, entre los que destacan los realizados por Close et al. (2005) y Schmal et al. (2011) demuestran que la técnica o régimen de fertilización exponencial es una práctica que no solo favorece un crecimiento superior, sino que también promueve la acumulación de reservas nutrimentales, en este estudio no ocurrió de esta manera. De acuerdo con los resultados, en plantas de E. cyclocarpum, el suministro de nutrimentos en forma exponencial no promueve características que podrían ser adecuadas para que las plantas se desempeñen satisfactoriamente en sitios competitivos. En la mayoría de los indicadores que relacionan a valores más elevados con mejor calidad de planta (altura, diámetro, peso seco aéreo y radical, índice de calidad de Dickson et al. y contenido de clorofila), la fertilización convencional resultó eficaz. Asimismo, los contenidos de nitrógeno y fósforo, y las respuestas de crecimiento en diámetro obtenidas en el ensayo de prueba de calidad en tezontle hacen patente lo anterior.

Considerando lo que establecen Oliet et al. (2013), es posible que por los diferentes patrones de crecimiento entre especies, los efectos del régimen de fertilización exponencial no siempre son favorables; sin embargo, tal hipótesis es controversial ya que Timmer (1997) indica que una de las principales ventajas de esta técnica es que se ajusta adecuadamente al crecimiento de las plantas para suministrar la cantidad de nutrimentos requeridos en cada etapa.

En otros trabajos, como los realizados por Schott et al (2013) y Everett et al. (2007), la adición de fertilizante en forma exponencial tampoco promovió un efecto favorable, pues cuando dichos autores examinaron los efectos de regímenes convencionales contra exponenciales en plantas de Populus tremuloides Michx., Pseudotsuga menziesii var. Glauca (Mirb.) Franco y Tsuga heterophylla (Raf.) Sarg., respectivamente, estas respondieron mejor a la fertilización convencional.

Estudiando la misma especie que Everett et al. (2007), Burgess (1991) atribuye el comportamiento a que especies con crecimiento rápido no se benefician completamente por dicho régimen de fertilización, principalmente porque las tasas de adición de fertilizante durante las fases iniciales de crecimiento pueden ser bajas e insuficientes para los requerimientos de las plantas.

Aunque tal autor basa sus conclusiones en coníferas, tal hipótesis podría extrapolarse a E. cyclocarpum, ya que en efecto, en comparación con el régimen convencional, las dosis de fertilizante suministrado durante las fases iniciales con el régimen exponencial fueron bajas, y porque también esta especie presenta un crecimiento rápido durante su fase de producción en vivero (Ngulube 1989).

\section{CONCLUSIONES}

La poda aérea no presenta un efecto favorable en la mayoría de los indicadores de calidad evaluados; sin embargo, dicha práctica propicia un crecimiento más equilibrado; es decir, las plantas podadas, especialmente al $25 \%$, muestran mejor relación entre la parte aérea y radical y son más robustas.

Los regímenes de fertilización tradicional y exponencial con sus respectivas dosis de fertilizante suministrado, no favorecen la calidad de la planta producida. En cambio, la fertilización convencional es la que más favorece este aspecto.

Ante un ambiente controlado de escasa o nula disponibilidad de nutrientes, las plantas producidas empleando el régimen de fertilización convencional especificado en este estudio, alcanzan las reservas nutrimentales necesarias para desempeñarse adecuadamente durante tres meses, en términos de supervivencia y crecimiento, en esta condición. Con lo anterior, se establecen pautas iniciales para producir en vivero planta de calidad de E. cyclocarpum; no obstante, se recomiendan trabajos en campo para dilucidar estas respuestas.

\section{AGRADECIMIENTOS}

Los autores expresamos nuestro total agradecimiento a la M. en C. Celi Gloria Calixto Valencia por el apoyo técnico que brindó a este trabajo.

\section{REFERENCIAS}

Aldana BR, MA Rodríguez. 2003. Procedimientos y cálculos básicos, útiles en la operación de viveros que producen en contenedor. México DF, México. Programa Nacional de Reforestación. Comisión Nacional Forestal. 46 p.

Berendse F, H De Kroon, WG Braakhekke. 2007. Acquisition, use and loss of nutrients In Pugnaire FI, F Valladares eds. 
Functional Plant Ecology 2nd. ed. Boca Raton, FL, USA. CRC Press. p. 259-283.

Burgess D. 1991. Western hemlock and Douglas-fir seedling development with exponental rates of nutrient addition. Forest Science 37: 54-57.

Cetina AVM, VAG Hernández, JJV Hernández. 1999. El manejo en vivero de Pinus greggii Engelm. y la calidad de planta. Agrociencia 33: 423-430.

Cetina AVM, VAG Hernández, MLO Delgado, JJV Hernández, AV Monter. 2002. Supervivencia y crecimiento en campo de Pinus greggii Engelm. previamente sometido a podas o sequia en vivero. Agrociencia 36: 233-241.

Cline MG, CA Harrington. 2007. Apical dominance and apical control in multiple flushing of temperate woody species. Canadian Journal of Forest Research 37: 74-83.

Close DC, I Bail, S Hunter, CL Beadle. 2005. Effects of exponential nutrient-loading on morphological and nitrogen characteristics and on after-planting performance of Eucalyptus globulus seedlings. Forest Ecology and Management 205: 397-403.

Di Rienzo JA, F Casanoves, MG Balzarini, L González, M Tablada, CW Robledo. 2012. InfoStat versión 2012. Grupo InfoStat, FCA, Universidad Nacional de Córdoba, Argentina. URL: http://www.infostat.com.ar

Donoso PJ, DP Soto, V Gerding. 2009. Efectos de la poda de tallo y fertilización de liberación controlada en vivero sobre el comportamiento de plántulas de Nothofagus nervosa en terreno. Bosque 30(1): 48-53.

Everett KT, BJ Hawkins, S Kiiskila. 2007. Growth and nutrient dynamics of Douglas-fir seedlings raised with exponential or conventional fertilization and planted with or without fertilizer. Canadian Journal of Forest Research 37: 25522562.

Grossnickle SC. 2012. Why seedlings survive: influence of plant attributes. New Forests 43: 711-738.

Hawkins BJ, G Henry. 1999. Nutrition and bud removal affect biomass and nutrient allocation in Douglas-fir and western red cedar. Tree Physiology 19 (3): 197-203.

Ibrahim M, P Zapata. 2012. Producción de madera en sistemas silvopastoriles. In Detlefsen G, E Somarriba eds. Producción de madera en sistemas agroforestales de Centroamérica. Turrialba, Costa Rica. Centro Agronómico Tropical de Investigación y Enseñanza. p.112-132.

Jobidon R, V Roy, G Cyr. 2003. Net effect of competing vegeta- tion on selected environmental conditions and performance of four spruce seedling stock sizes after eight years in Québec (Canada). Annals of Forest Science 60(7): 691-699.

Johnson JD , ML Cline. 1991. Seedling quality of Southern Pines. In Duryea ML, PM Dougherty eds. Forest Regeneration Manual. Netherlands. Kluwer Academic Publishers. p. 143-159.

Landis TD. 1989. Mineral nutrients and fertilization. In Landis TD, RW Tinus, SE McDonald, JP Barnett eds. The container tree nursery manual Vol. 4 Agric. Handbook 674. U.S. Washington D.C., USA. U.S. Department of Agriculture, Forest Service. p. 1-67.

Mc Nabb K, C Vanderschaaf. 2005. Growth of graded sweetgum 3 years after root and shoot pruning. New Forests 29: 313320.

Ngulube MR. 1989. Seed germination, seedling growth and biomass production of eigth Central-American multipurpose trees under nursery conditions in Zomba, Malawi. Forest Ecology and Management 27: 21-27.

Oliet JA, J Puértolas, R Planelles, DF Jacobs. 2013. Nutrient loading of forest tree seedlings to promote stress resistance and field performance: a Mediterranean perspective. New Forests 44: 649-669.

Schmal JL, DF Jacobs, CO’ Reilly. 2011. Nitrogen budgeting and quality of exponentially fertilized Quercus robur seedlings in Ireland. European Journal of Forest Research 130: 557-567.

Schott KM, BD Pinno, SM Landhäusser. 2013. Premature shoot growth termination allows nutrient loading of seedlings with an indeterminate growth strategy. New Forests 44: 636-647.

Timmer VR. 1997. Exponential nutrient loading: a new fertilization technique to improve seedling performance on competitive sites. New Forests 13: 279-299.

Villar SP, J Puértolas, B Cuesta, JL Peñuelas, M Uscola, NH Guerrer, JMB Rey. 2012. Increase in size and nitrogen concentration enhances seedling survival in Mediterranean plantations. Insights from an ecophysiological conceptual model of plant survival. New Forests 43: 755-770.

Villar SP, JL Puértolas, JLN Peragón, LF Benito, SD Lerena. 2013. Is nitrogen fertilization in the nursery a suitable tool for enhancing the performance of Mediterranean oak plantations? New Forests DOI: 10.1007/s11056-013-9374-8.

Wilson BF. 2000. Apical control of branch growth and angle in woody plants. American Journal of Botany 87(5): 601-607. 
